\title{
Three-Dimensional Determination of the Coordinates of Individual Atoms in Materials
}

Jianwei Miao ${ }^{1 *}$, Rui Xu${ }^{1}$, Chien-Chun Chen ${ }^{1 \dagger}$, Li Wu${ }^{1}$, M. C. Scott ${ }^{1}$, W. Theis ${ }^{2}$, Colin Ophus $^{3}$, Matthias Bartels ${ }^{1}$, Yongsoo Yang ${ }^{1}$, Hadi Ramezani-Dakhel ${ }^{4}$, Michael R. Sawaya ${ }^{5}$, Hendrik Heinz ${ }^{4}$, Laurence D. Marks $^{6}$ and Peter Ercius ${ }^{3}$

1. Department of Physics \& Astronomy and California NanoSystems Institute, and University of California, Los Angeles, CA, USA.

2. Nanoscale Physics Research Laboratory, School of Physics and Astronomy, University of Birmingham, Edgbaston, Birmingham, UK.

3. National Center for Electron Microscopy, Molecular Foundry, Lawrence Berkeley National Laboratory, Berkeley, California, USA.

4. Department of Polymer Engineering, University of Akron, Akron, Ohio, USA.

${ }^{5 .}$ Howard Hughes Medical Institute, UCLA-DOE Institute of Genomics and Proteomics, Los Angeles, California, USA.

6. Department of Materials Science and Engineering, Northwestern University, Evanston, IL, USA.

†Present address: Department of Physics, National Sun Yat-sen University, Kaohsiung, Taiwan.

In 1959, Richard Feynman challenged the electron microscopy community to locate the positions of individual atoms in substances (1). Over the last fifty-five years, significant advances have been made in electron microscopy. With the development of aberration-corrected electron optics (2,3), twodimensional transmission electron microscopy (TEM) imaging has reached sub- $0.5 \AA$ resolution (4). Using scanning TEM (STEM) (5-6) and a three-dimensional (3D) image reconstruction method known as equal slope tomography (EST) $(8,9)$, electron tomography has achieved $2.4 \AA$ resolution and was applied to image the $3 \mathrm{D}$ core structure of edge and screw dislocations at atomic resolution $(10,11)$. More recently, TEM has been used to determine the 3D atomic structure of gold nanoparticles by averaging 939 particles (12). Notwithstanding these important developments, however, Feynman's 1959 challenge - 3D localization of the positions of atoms in a substance without using averaging or a priori knowledge of sample crystallinity - remains elusive. Here, we report the determination of the 3D positions of 3,769 individual atoms in a tungsten needle sample with a precision of $\sim 19$ picometers and the identification of a point defect inside the sample in three dimensions (13). Our method consists of acquisition of a tilt series with an aberration-corrected STEM, 3D EST reconstructions of the tilt series, tracing individual atoms from the reconstructions, and 3D atomic model refinement, in which the crystallinity of the sample is not assumed. By comparing the positions of individual atoms with a body-centred-cubic (bcc) crystal lattice, we determine the atomic displacement field and the full strain tensor with a 3D resolution of $\sim 1 \mathrm{~nm}^{3}$ and a precision of $\sim 10^{-3}$, which are further verified by density functional theory calculations and molecular dynamics simulations (13). The ability to precisely localize the 3D positions of individual atoms in materials without assuming crystallinity, measure point defects, and determine the 3D atomic displacement field and the full strain tensor is expected to find important applications in both fundamental and applied sciences. [14] 


\section{References:}

[1] Feynman, R. P. in Feynman and Computation (ed. Hey, J. G. ) 63-76 (Perseus Press, Cambridge, Massachusetts, 1999).

[2] Haider, M., et al. Nature 392, 768-769 (1998).

[3] Batson, P. E., Dellby, N. \& Krivanek, O. L. Nature 418, 617-620 (2002).

[4] Erni, R. et al, Phys. Rev. Lett. 102, 096101(2009).

[5] Midgley, P. A. \& Weyland, M. STEM Tomography. In Scanning Transmission Electron

Microscopy: Imaging and Analysis. ed. Pennycook, S. J. \& Nellist, P. D. pp. 353-392 (Springer, 2011).

[6] LeBeau, J. M. et al, Phys. Rev. Lett. 100, 206101 (2008)

[7] D. A. Muller. Nature Materials 8, 263-270 (2009).

[8] Miao, J., Föster, F. \& Levi, O. Phys. Rev. B 72, 052103 (2005).

[9] Zhao, Y. et al. Proc. Natl. Acad. Sci. USA 109, 18290-18294 (2012).

[10] Scott, M. C. et al. Nature 483, 444-447 (2012).

[11] Chen, C.-C. et al. Nature 496, 74-77 (2013).

[12] Azubel, M. et al. Science 345, 909-912 (2014).

[13] Xu, R. et al. Nature Mater. 14, 1099-1103 (2015).

[14] This work was primarily supported by the Office of Basic Energy Sciences of the U.S. Department of Energy (Grant No. DE-SC0010378). ADF-STEM imaging was performed on TEAM I at the

Molecular Foundry, which is supported by the Office of Science, Office of Basic Energy Sciences of the U.S. Department of Energy under Contract No. DE-AC02—05CH11231.

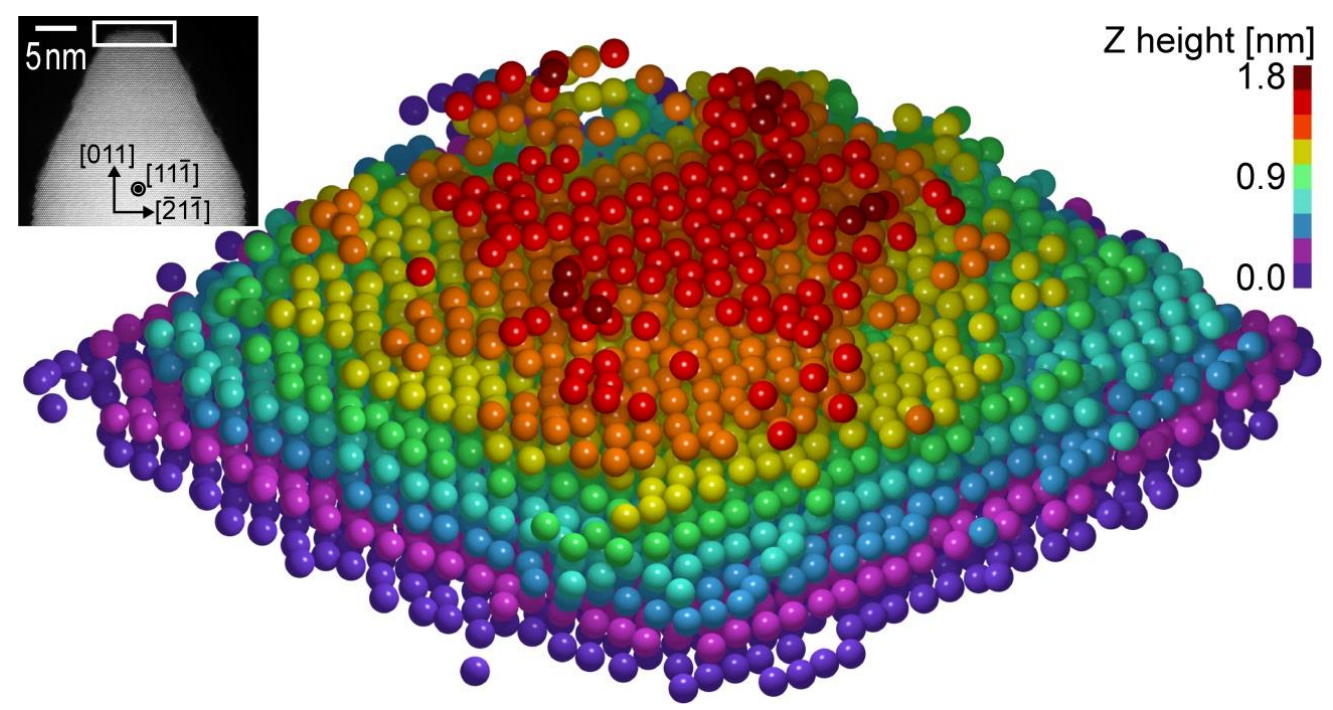

Figure 1. 3D positions of individual atoms in a tungsten needle sample revealed by electron tomography. The experiment was conducted using an aberration-corrected STEM. A tilt series of 62 projections was acquired from the sample by rotating it around the [011] axis. The inset shows a representative projection at $0^{\circ}$. After post-processing, the apex of the sample (labelled with a rectangle in the inset) was reconstructed by the EST method. The 3D positions of individual atoms were then traced from the reconstructions and refined using the 62 experimental projections. The $3 \mathrm{D}$ atomic model of the sample consists of 9 atomic layers along the [011] direction, labelled with crimson (dark red), red, orange, yellow, green, cyan, blue, magenta and purple from layers 1 to 9, respectively. (Ref. 13) 